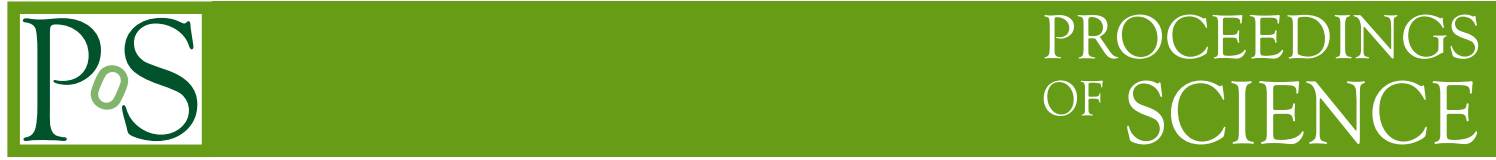

\title{
2D Gauge Field Theory
}

\section{Andrey V. Koshelkin*}

Moscow Institute for Physics and Engineering, Kashirskoye sh., 31, 115409 Moscow, Russia

E-mail: A Kosh@internets.ru

\section{Cheuk-Yin Wong}

Physics Division, Oak Ridge National Laboratory, Oak Ridge, TN 37831

E-mail: wongc@ornl.gov

We show from the action integral that under the assumption of longitudinal dominance and transverse confinement, QCD4 in $(3+1)$ dimensional space-time can be approximately compactified into QCD2 in $(1+1)$ dimensional space-time. In such a process, we find the relation between the coupling constant $g(2 D)$ in QCD2 and the coupling constant $g(4 D)$ in QCD4. We also show that quarks and gluons in QCD2 acquire masses as a result of the compactification.

Sixth International Conference on Quarks and Nuclear Physics,

April 16-20, 2012

Ecole Polytechnique, Palaiseau, Paris

\footnotetext{
* Speaker.
} 


\section{Introduction}

Previously, t'Hooft showed that in the limit of large $N_{c}$ with fixed $g^{2} N_{c}$ in single-flavor quantum chromodynamics in $(3+1)$ dimensional space-time (QCD4), planar diagrams with quarks at the edges dominate, whereas diagrams with the topology of a fermion loop or a wormhole are associated with suppressing factors of $1 / N_{c}$ and $1 / N_{c}^{2}$, respectively [1]. In this case a simple-minded perturbation expansion with respect to the coupling constant $g$ cannot describe the spectrum, while the $1 / N_{c}$ expansion may be a reasonable concept, in spite of the fact that $N_{c}$ is equal to 3 and is not very big. The dominance of the planar diagrams allows one to consider QCD in one space and one time dimensions (QCD2) and the physics resembles those of the dual string or a flux tube, with the physical spectrum of a straight Regge trajectory [2]. The properties of QCD in two-dimensional space-time have been investigated by many workers [1, 2, 3]. The flux tube picture of longitudinal dynamics manifests itself in various aspects of hadron spectroscopy [4].

In the high-energy arena, the flux tube picture finds phenomenological applications in hadron collisions and high-energy $e^{-} e^{+}$annihilations [5, 6, 7, 8]. In these high-energy processes, the (average) transverse hadron momenta of produced hadrons are observed to be limited, as appropriate for particles confined in a flux tube. The idealization of the three-dimensional flux tube as a one-dimensional string leads to the string fragmentation picture of particle production in $(1+1)$ space-time dimensions. The classical Lund model of string fragmentation has been quite successful in describing particle production processes in hadron collisions and $e^{-} e^{+}$annihilations at high energies [5, 7].

With the successes of lower-dimensional descriptions of high-energy collision processes in QCD, we would like to examine the circumstances in high-energy processes under which QCD4 in $(3+1)$ dimensions can be compactified into QCD2 in (1+1) dimensions, if one starts with the QCD4 action integral. In such a process, we will be able to find out how quantities in the compactified QCD2 can be related to quantities in QCD4. The success of the compactification program will facilitate the examination of some problems in QCD4 in the simpler dynamics of QCD2.

\section{2. $4 D \rightarrow 2 D$ Compactification in the Action Integral}

The $4 D$-action $\mathscr{A}(4 D)$ resides in $(3+1)$ dimensional space-time. There are however environments which allow the compactification of the $4 D$ action to reside in two-dimensional $(1+1)$ space-time, within which the dynamics can be greatly simplified.

We note that in hadron collisions and $e^{-} e^{+}$annihilations at high energies, the string fragmentation process occurs when a valence quark pulls apart from a valence antiquark longitudinally at high energies. It is therefore reasonable to conceive that the QCD4 compactification can take place under the dominance of longitudinal dynamics, not only of the leading valence quark and antiquark pair, but also the produced $q \bar{q}$ parton pairs. In the Lorentz gauge, as $A_{v}$ is proportional to the current $j_{v}$, the gauge field components $A_{1}^{a}$ and $A_{2}^{a}$ along the transverse direction are then small in magnitude in comparison with those of $A_{0}^{a}$ and $A_{3}^{a}$ and can be neglected. The absence of the transverse gauge fields provides a needed simplification for compactification.

The spatially one-dimensional string being an idealization of a more realistic three-dimensional flux-tube, the description of produced $q \bar{q}$ parton pairs within the string presumes the confinement 
of these produced partons inside the string. Hence, it is reasonable to conceive further that the QCD4 compactification takes place under transverse confinement. We can describe transverse confinement in terms of a confining scalar interaction $S\left(\mathbf{r}_{\perp}\right)$ and the quark mass function is then $m\left(\mathbf{r}_{\perp}\right)=m_{0}+S\left(\mathbf{r}_{\perp}\right)$, where $m_{0}$ is the quark rest mass.

Therefore, under the assumption of longitudinal dominance and transverse confinement, the $\mathrm{SU}(\mathrm{N})$ gauge invariant action integral in $(3+1)$ Minkowski space-time is given by [9]:

$$
\begin{gathered}
\mathscr{A}(4 D)=\int d^{4} x\left\{\operatorname{Tr}\left[\bar{\Psi}(4 D, x)\left(\gamma^{\mu}(4 D)\left(i \partial_{\mu}+g(4 D) T_{a} A_{\mu}^{a}(4 D, x)\right)-m\left(\mathbf{r}_{\perp}\right)\right) \Psi(4 D, x)\right]\right. \\
\left.-\frac{1}{16 \pi} F_{\mu \nu}^{a}(4 D, x) F_{a}^{\mu v}(4 D, x)\right\} \\
F_{\mu \nu}^{a}(4 D, x)=\partial_{\mu} A_{v}^{a}(4 D, x)-\partial_{v} A_{\mu}^{a}(4 D, x)+g(4 D) f_{b c}^{a} A_{\mu}^{b}(4 D, x) A_{v}^{c}(4 D, x),
\end{gathered}
$$

where $A_{\mu}^{a}(4 D, x)$ and $\Psi(4 D, x)$ are gauge and fermion fields respectively with coordinates $x \equiv x^{\mu}=$ $\left(x^{0}, \mathbf{x}\right)=\left(x^{0}, x^{1}, x^{2}, x^{3}\right)$ and transverse coordinates $\mathbf{r}_{\perp}=\left(x^{1}, x^{2}\right), g=g(4 D)$ is the coupling constant, $\gamma^{\nu}(4 D)$ are Dirac matrices, and $T_{a}$ are the generators of the $\mathrm{SU}(\mathrm{N})$ group.

\subsection{Fermion part of the action integral}

We first examine $\mathscr{A}(4 D, F)$, the part of the $4 D$ action integral in Eq. (2.1) that involves the fermion field. To carry out the compactification, we write the Dirac fermion field $\Psi(4 D, x)$ in terms of functions $G_{ \pm}\left(\vec{r}_{\perp}\right)$ and $f_{ \pm}\left(x^{0}, x^{3}\right)[\overline{6}]$ :

$$
\Psi(4 D, x)=\frac{1}{\sqrt{2}}\left(\begin{array}{c}
G_{1}\left(\mathbf{r}_{\perp}\right)\left(f_{+}\left(x^{0} ; x^{3}\right)+f_{-}\left(x^{0} ; x^{3}\right)\right) \\
-G_{2}\left(\mathbf{r}_{\perp}\right)\left(f_{+}\left(x^{0} ; x^{3}\right)-f_{-}\left(x^{0} ; x^{3}\right)\right) \\
G_{1}\left(\mathbf{r}_{\perp}\right)\left(f_{+}\left(x^{0} ; x^{3}\right)-f_{-}\left(x^{0} ; x^{3}\right)\right) \\
G_{2}\left(\mathbf{r}_{\perp}\right)\left(f_{+}\left(x^{0} ; x^{3}\right)+f_{-}\left(x^{0} ; x^{3}\right)\right)
\end{array}\right) .
$$

Using this explicit form of the Dirac fermion field $\Psi(4 D, x)$, we carry out the simplifications and integrations over $x^{1}$ and $x^{2}$ that eventually lead from $\mathscr{A}(4 D, F)$ to $\mathscr{A}(2 D, F)$,

$$
\mathscr{A}(2 D, F)=\operatorname{Tr} \int d^{2} X \bar{\Psi}(2 D, X)\left[\left(i \gamma^{\mu}(2 D) \partial_{\mu}+g(2 D) \gamma^{\mu} T_{a} A_{\mu}^{a}(2 D, X)\right)-m_{q T}\right] \Psi(2 D, X),
$$

where $\mu=0,3$, and we have introduced the Dirac fermion field $\Psi(2 D), \gamma$-matrices, and metric tensor $g_{\mu \nu}$, according to the following specifications in (1+1)-dimensional space-time in QCD2,

$$
\begin{aligned}
& \Psi(2 D, X)=\left(\begin{array}{c}
f_{+}(X) \\
f_{-}(X)
\end{array}\right), \quad X=\left(x^{0} ; x^{3}\right), \\
& \gamma^{0}(2 D)=\left(\begin{array}{cc}
1 & 0 \\
0 & -1
\end{array}\right), \quad \gamma^{3}(2 D)=\left(\begin{array}{cc}
0 & 1 \\
-1 & 0
\end{array}\right), \quad g_{\mu \nu}(2 D)=\left(\begin{array}{cc}
1 & 0 \\
0 & -1
\end{array}\right) .
\end{aligned}
$$

The $2 D$ coupling constant $g(2 D)$ is related to $4 D$ coupling constant $g(4 D)$ by

$$
g(2 D)=\int d x^{1} d x^{2} g(4 D)\left[\left|G_{1}\left(\mathbf{r}_{\perp}\right)\right|^{2}+\left|G_{2}\left(\mathbf{r}_{\perp}\right)\right|^{2}\right]^{3 / 2},
$$


where the transverse wave functions $G_{1,2}\left(\mathbf{r}_{\perp}\right)$ are normalized according to

$$
\int d x^{1} d x^{2}\left(\left|G_{1}\left(\mathbf{r}_{\perp}\right)\right|^{2}+\left|G_{2}\left(\mathbf{r}_{\perp}\right)\right|^{2}\right)=1 .
$$

The transverse quark mass $m_{q T}$ in Eq. (2.4) is given by

$$
m_{q T}=\int d x^{1} d x^{2}\left\{m\left(\mathbf{r}_{\perp}\right)\left(\left|G_{1}\left(\mathbf{r}_{\perp}\right)\right|^{2}-\left|G_{2}\left(\mathbf{r}_{\perp}\right)\right|^{2}\right)+\left[\left(G_{1}^{*}\left(\mathbf{r}_{\perp}\right)\left(p_{1}-i p_{2}\right) G_{2}\left(\mathbf{r}_{\perp}\right)\right)-\text { h.c. }\right]\right\} .
$$

In obtaining these results, we have considered $2 D$ gauge fields $A_{\mu}^{a}\left(2 D, x^{0}, x^{3}\right)$ related to the $4 D$-field gauge fields $A_{\mu}^{a}\left(4 D, x^{0}, x^{3}, \mathbf{r}_{\perp}\right)$ by

$$
A_{\mu}^{a}\left(4 D, x^{0}, x^{3}, \mathbf{r}_{\perp}\right)=\sqrt{\left|G_{1}\left(\mathbf{r}_{\perp}\right)\right|^{2}+\left|G_{2}\left(\mathbf{r}_{\perp}\right)\right|^{2}} A_{\mu}^{a}\left(2 D, x^{0}, x^{3}\right), \quad \mu=0,3 .
$$

The above equation means that along with the confinement of the fermions, for which the wave function $G_{1,2}\left(\mathbf{r}_{\perp}\right)$ is confined within a finite region of transverse coordinates $\mathbf{r}_{\perp}$, we also consider the confinement of the gauge field $A_{\mu}^{a}(4 D, x), \mu=0,3$, within the same finite region of transverse coordinates, as in the case for a flux tube.

The result of Eq. (2.7) reveals that as a result of the compactification of QCD4, the coupling constant $g(2 D)$ in lower dimensional space in QCD2 acquires the dimension of a mass, and is related to the confining wave functions of the fermions. Fermions in different excited states inside the tube will have different coupling constants as indicated in Eq. (2.7). The effective quark mass $m_{q T}$ also depends on the transverse fermion wave functions, as indicated in Eq. (2.9). In the lower two-dimensional space-time, fermions in excited transverse states have a quark mass different from those in the ground transverse states.

\subsection{Gauge field part of the action integral}

To go from $\mathscr{A}(4 D, F)$ to $\mathscr{A}(2 D, F)$ we have assumed that the currents in the $x^{0}$ and $x^{3}$ directions are much larger in magnitude than the currents in the transverse directions so that $A_{1}^{a}$ and $A_{2}^{a}$ are small in comparison and can be neglected. As a consequence, $F_{12}(4 D)=0$ (we omit the superscript symbol $a$ (color) for simplicity). The evaluation of all other components of $F_{\mu v}$ give for the gauge field part of the action integral

$$
\begin{aligned}
& \int \frac{d^{4} x}{16 \pi} F_{\mu v}^{a}(4 D) F_{a}^{\mu v}(4 D)=\int \frac{d x^{0} d x^{3}}{16 \pi} \int d x^{1} d x^{2}\left(\left|G_{1}\left(\mathbf{r}_{\perp}\right)\right|^{2}+\left|G_{2}\left(\mathbf{r}_{\perp}\right)\right|^{2}\right) F_{03}^{a}(2 D) F_{a}^{03}(2 D) \\
&-\int \frac{d x^{0} d x^{3}}{16 \pi} \int d x^{1} d x^{2}\left(\left\{\partial_{1}\left[\left|G_{1}\left(\mathbf{r}_{\perp}\right)\right|^{2}+\left|G_{2}\left(\mathbf{r}_{\perp}\right)\right|^{2}\right]^{1 / 2}\right\}^{2}+\left\{\partial_{2}\left[\left|G_{1}\left(\mathbf{r}_{\perp}\right)\right|^{2}+\left|G_{2}\left(\mathbf{r}_{\perp}\right)\right|^{2}\right]^{1 / 2}\right\}^{2}\right) \\
& \times\left[A_{0}\left(2 D, x^{0}, x^{3}\right) A^{0}\left(2 D, x^{0}, x^{3}\right)+A_{3}\left(2 D, x^{0}, x^{3}\right) A^{3}\left(2 D, x^{0}, x^{3}\right)\right] .
\end{aligned}
$$

It is useful to introduce the gluon mass $m_{g T}$ that arises from the confinement of the gluons in the transverse direction,

$$
m_{g T}^{2}=\frac{1}{2} \int d x^{1} d x^{2}\left[\left\{\partial_{1}\left(\sum_{i=1}^{2}\left|G_{i}\left(\vec{r}_{\perp}\right)\right|^{2}\right)^{1 / 2}\right\}^{2}+\left\{\partial_{2}\left(\sum_{i=1}^{2}\left|G_{i}\left(\vec{r}_{\perp}\right)\right|^{2}\right)^{1 / 2}\right\}^{2}\right] .
$$


As this gluon mass $m_{g T}$ arises from the confinement compactification of the gluon within the flux tube, we can call such a mass the compactification mass of the gluon. Equation (2.11) becomes

$$
\int \frac{d^{4} x}{16 \pi} F_{\mu v}^{a}(4 D) F_{a}^{\mu v}(4 D)=\int \frac{d x^{0} d x^{3}}{16 \pi}\left\{F_{03}^{a}(2 D) F_{a}^{03}(2 D)-2 m_{g T}^{2}\left[A_{\mu}^{a}(2 D) A_{a}^{\mu}(2 D)\right]\right\}
$$

We collect all the fermion and gauge field parts of the action integral in $\mathscr{A}(4 D)$ in Eq. (2.1). All terms in the action integral $\mathscr{A}(4 D)$ (including matrices and coefficients) are in the Minkowski $(1+1)$ dimensional space-time. We can rename the action integral $\mathscr{A}(4 D)$ to be $\mathscr{A}(2 D)$ given explicitly by

$$
\begin{gathered}
\mathscr{A}(2 D)=\int d^{2} X\left\{\operatorname { T r } \left[\bar{\Psi}(2 D, X)\left[\gamma^{k}(2 D)\left(i \partial_{\mu}+g(2 D) T_{a} A_{\mu}^{a}(2 D, x)\right)-m_{q T}\right] \Psi(2 D, X)\right.\right. \\
\left.-\frac{1}{16 \pi} F_{\mu \nu}^{a}(2 D) F_{a}^{\mu v}(2 D)+\frac{1}{8 \pi} m_{g T}^{2}\left[A_{a}^{\mu}(2 D) A_{\mu}^{a}(2 D)\right]\right\} .
\end{gathered}
$$

Thus, in the presence of longitudinal dominance and transverse confinement, we succeed in compactifying the action integral from $\mathscr{A}(4 D)$ in QCD4 to $\mathscr{A}(2 D)$ in QCD2, by introducing $g_{2 D}, m_{q T}$, and $m_{g T}$ that contain information about the transverse profile. All the transverse flux tube information is subsumed under these quantities. In this way, the $2 D$ gauge field appears to be massive where $m_{g T}$ and $m_{g T}$ arise as a consequence of the transverse confining motion of both fermion and gauge fields. The physical explanation of such effect consists in the decrease of the number of trajectories in moving from one point of the space to another point, as a direct consequence of compactification. Such constraints in movement manifest themselves as masses of field particles. The magnitudes of $m_{q T}$ and $m_{g T}$ depend strongly on the kind of the compactification that is dictated by the transverse functions $G_{1}\left(\mathbf{r}_{\perp}\right)$ and $G_{2}\left(\mathbf{r}_{\perp}\right)$ (see Eqs.(2.9) and (2.12)).

\section{Equations of a transverse motion in a tube and Fermion effective mass}

To obtain the equations of motion for the functions $G_{1}\left(\vec{r}_{\perp}\right)$ and $G_{2}\left(\vec{r}_{\perp}\right)$, we vary the action integral $\mathscr{A}(4 D)(2.1)$ with the fermion fields $\Psi(4 D, x)$ given by Eq.(9), under the constraint of the normalization condition, (2.8). To do this we construct a new functional $\mathscr{F}$

$$
\mathscr{F}=\mathscr{A}(4 D)+\frac{\lambda}{2} \int d x^{1} d x^{2}\left(\sum_{i=1}^{2}\left|G_{i}\left(\vec{r}_{\perp}\right)\right|^{2}\right) \int d x^{0} d x^{3}\left(\bar{\psi}(2 D, X) m_{q T} \psi(2 D, X)\right),
$$

where $\lambda$ is the Lagrange multiplier. The last term in Eq. (3.1) takes into account the unitarity of a fermion field in the 4D space-time. Varying the last equation with respect to the functions $G_{1}\left(\vec{r}_{\perp}\right)$ and $G_{2}\left(\vec{r}_{\perp}\right)$ we derive

$$
\begin{aligned}
& \left(p_{1}+i p_{2}\right) G_{1}\left(\vec{r}_{\perp}\right)=\left(m\left(\vec{r}_{\perp}\right)+\lambda\right) G_{2}\left(\vec{r}_{\perp}\right), \quad\left(p_{1}-i p_{2}\right) G_{2}\left(\vec{r}_{\perp}\right)=\left(\lambda-m\left(\vec{r}_{\perp}\right)\right) G_{1}(\vec{r}), \\
& \left(p_{1}+i p_{2}\right) G_{2}^{*}\left(\vec{r}_{\perp}\right)=\left(m\left(\vec{r}_{\perp}\right)-\lambda\right) G_{1}^{*}\left(\vec{r}_{\perp}\right), \quad\left(p_{1}-i p_{2}\right) G_{1}^{*}\left(\vec{r}_{\perp}\right)=-\left(m\left(\vec{r}_{\perp}\right)+\lambda\right) G_{2}^{*}(\vec{r}) .
\end{aligned}
$$

Carrying out complex conjugation in the last two equations we obtain $\lambda=\lambda^{*}$. Substituting the equations (3.2) for $G_{1,2}\left(\vec{r}_{\perp}\right)$ functions into the formula (2.9) for $m_{q T}$ we get

$$
m_{q T}=\lambda .
$$


Thus, the effective mass of the compactified 2D fermion field is equal to the energy of the transverse motion of the 4D fermion, which is an eigenvalue of Eq. (3.2). We should note here that the 2D fermion can generally gain a mass even when the initial 4D fermion appears to be massless. The explanation of such phenomenon is the same as before. The compactification effectively leads to constraints in moving a fermion from one point of a space-time to another one due to the decrease of the number of trajectories in the 2D space-time, as compared to the 4D space-time.

\section{Conclusion}

Under the assumption of longitudinal dominance and transverse confinement, the SU(N) gauge invariant field theory of QCD4 can be compactified into QCD2 in Minkowski $(1+1)$ dimensional space-time from the consideration of the action integral. The compactified 2D action integral $\mathscr{A}(2 D)$ depends only on $2 \mathrm{D}$-fields. The corresponding coupling constants, effective quark, and gluon masses in two-dimensional space-time are derived. Such compactification leads to strong changes in physics of the 2D Lagrangian that is manifested in both the renormalization of coupling constant and fermions as well as gauge field bosons acquiring masses.

\section{Acknowledgment}

The research was supported in part by the Division of Nuclear Physics, U.S. Department of Energy.

\section{References}

[1] G. t'Hooft, Nucl. Phys. B72, 461 (1974).

[2] G. t'Hooft, Nucl. Phys. B75, 461 (1974).

[3] Y. Frishman and J. Sonnenschein, Phys. Rep. 223, 309 (1993); S. Dalley and I. R. Klebanov, Phys. Rev. D47, 2527 (1993); D. Kutasov and A. Schwimmer, Nucl. Phys. B442, 447 (1995); D. Gross, I. R. Klebanov, A. Matysin, and A. V. Smilga Nucl. Phys. B461, 109 (1996); E. Abdalla and M.C.B. Abdalla, Phys. Reports 265, 253 (1996); E. Witten, Commun. Math. Phy. 92, 455 (1984)

[4] N. Isgur and J. Paton, Phys. Rev. D31, 2910 (1985).

[5] B. Andersson, G. Gustafson, and T. Sjöstrand, Zeit. für Phys. C20, 317 (1983); B. Andersson, G. Gustafson, G. Ingelman, and T. Sjöstrand, Phys. Rep. 97, 31 (1983); T. Sjöstrand and M. Bengtsson, Computer Physics Comm. 43, 367 (1987); B. Andersson, G. Gustavson, and B. Nilsson-Alqvist, Nucl. Phys. B281, 289 (1987).

[6] C. Y. Wong, R. C. Wang, and C. C. Shih, Phys. Rev. D 44, 257 (1991).

[7] C. Y. Wong, Introduction to High-Energy Heavy-Ion Collisions, World Scientific Publisher, 1994.

[8] C. Y. Wong, Phys. Rev. C80, 054917 (2009).

[9] M. E. Peskin, D. V. Schroeder, An introduction to Quantum Field Theory, Addison-Wesley Publishing Company, 1995. 